\title{
Embarazo posterior a tratamiento conservador de teratoma inmaduro de ovario y comportamiento de los niveles de alfa-fetoproteína
}

\author{
Alma Villarreal-Barranca ${ }^{1}$, Ursula Torres-Herrera², Melissa Campos-Zamora ${ }^{2}$, Elsa Moreno-Verduzco ${ }^{3}$ \\ Salvador Espino-Sosa ${ }^{4}$, Enrique Reyes-Muñoz ${ }^{5 *}$, Teresa M. Morales-Montiel ${ }^{6}$ y \\ Miguel A. Morales-Palomares ${ }^{7}$
}

${ }^{1}$ Facultad de Medicina, Universidad Veracruzana, Verazcruz; ${ }^{2}$ Ginecología y Obstetricia, Instituto Nacional de Perinatología Isidro Espinosa de los Reyes, Ciudad de México; ${ }^{3}$ Subdirección de Auxiliares de Diagnóstico, Instituto Nacional de Perinatología Isidro Espinosa de los Reyes, Ciudad de México; ${ }^{4}$ Subdirección de Investigación Clínica, Instituto Nacional de Perinatología Isidro Espinosa de los Reyes, Ciudad de México; ${ }^{5}$ Coordinación de Endocrinología, Instituto Nacional de Perinatología Isidro Espinosa de los Reyes, Ciudad de México; 'Unidad Médica de Alta Especialidad, Hospital de Gineco-Obstetricia No. 3, Centro Médico Nacional (CMN) La Raza, Instituto Mexicano del Seguro Social, Ciudad de México; PCirugia Oncológica, Hospital General de México, Ciudad de México. México

\section{Resumen}

Antecedentes: El teratoma inmaduro de ovario es un tumor de células germinales maligno, ocurre típicamente en mujeres jóvenes y representa menos del $1 \%$ de todos los tumores de ovario. La literatura sobre el comportamiento de los niveles de alfa-fetoproteína previo, durante y posterior a un embarazo en mujeres con antecedente de teratoma inmaduro es limitada. Objetivo: Reportar el resultado del embarazo y el comportamiento de los niveles de alfa-fetoproteína en una mujer con̄ tecedente de teratoma inmaduro de ovario. Descripción del caso: Paciente de sexo femenino, de 32 años, con antecedênte de resección de teratoma inmaduro de ovario izquierdo y aplicación de quimioterapia con cisplatino, bleomicina y etopósido. Logró el embarazo espontáneo que concluyó con el nacimiento de un recién nacido de término sano. Los niveles de alfa-tetoproteína previo y posterior a la resección del teratoma, a la semanas de gestación 8, 17, 27, 31, 35, y a las 4 semanas posparto fueron: 41.2 y 2.1, 3.8, 34.6, 183.3, 209.5, 149.3, y $1.7 \mathrm{ng} / \mathrm{ml}$. Conclusiones: El embarazo posterior al manejo conservador de teratoma de ovario inmaduro puede ser espontáneo y exitoso. En el presente caso la alfa-fetoproteína se incrementó a partir del segundo trimestre, con un pico máximo en el tercer trimestre, y disminuyó a valores normales en el puerperio tardio.

Palabras clave: Teratoma inmaduro de ovario. Alfa-fetoproteína. Tratamiento conservador. Preservación de fertilidad.

\section{Pregnancy after conservative treatment of ovarian immature teratoma and levels of alpha-fetoprotein}

\section{Abstract}

Background: The immature ovarian teratoma is a malignant germ cell tumor that typically occurs in young women and represents $<1 \%$ of ovarian tumors. Currently there is limited availability of literature about of alpha-fetoprotein levels before, during and after pregnancy in women with a history of immature teratoma. Objective: Report the alpha-fetoprotein levels before, during and after pregnancy of a woman with history of ovarian immature teratoma. Case description: Female, $32 \overline{\mathrm{ye}}$ ars

\section{Correspondencia:}

*Enrique Reyes-Muñoz

E-mail: dr.enriquereyes@gmail.com

0187-5337/@ 2019. Instituto Nacional de Perinatología Isidro Espinosa de los Reyes. Publicado p licencia CC BY-NC-ND (http://creativecommons.org/licenses/by-nc-nd/4.0/).
Fecha de recepción: 26-02-2019

Fecha de aceptación: 15-03-2019

DOI: 10.24875/PER.19000014
Disponible en internet: $18-12-2019$ Perinatol Reprod Hum. 2019;33:80-83 www.perinatologia.mx 
old, with history of resected an immature teratoma of the left ovary plus 2 cycles of chemotherapy. Afterwards she presented a spontaneous pregnancy that concluded with the birth of a healthy newborn at term. Levels of alpha-fetoprotein before and after resection of teratoma, and in the weeks of gestation 8, 17,27,31, 35 and 4 weeks postpartum were: 41.2 and 2. 1, 3.8, 34.6, 183.3, 209.5, 149.3 and $1.7 \mathrm{ng} / \mathrm{mL}$. Conclusions: The pregnancy after management of immature teratoma can be succesful and spontaneous. In this case the levels of alpha-fetoprotein increased from the second trimester of pregnancy with a peak in the third trimester and decreased to normal values in the late postpartum period.

Key words: Ovarian immature teratoma. Alpha-fetoprotein. Conservative treatment. Fertility preservation.

\section{Antecedentes}

El teratoma inmaduro de ovario es un tumor de células germinales maligno, que ocurre con mayor frecuencia en las primeras dos décadas de la vida y representa el $1 \%$ del total de cáncer de ovario ${ }^{1}$. En México, la frecuencia de neoplasias malignas de ovario es del $3.4 \%$, y de ellas, el teratoma se presenta hasta en el $21 \%$ de los casos ${ }^{2}$.

Típicamente, el teratoma inmaduro está compuesto por tejido de 2 o 3 capas germinales: ectodermo, endodermo y mesodermo. Histológicamente, hay diversas cantidades de tejido inmaduro, casi siempre con diferenciación neural'.

Debido a la baja frecuencia de estos tumores, la información disponible acerca del tratamiento se limita a estudios clínicos y revisiones retrospectivas con pocas pacientes y que casi siempre combinan todos los tumores de células germinales. Se recomienda que, independientemente de la etapa clínica, el tratamiento inicial sea quirúrgico tomando en cuenta el deseo de preservación de la fertilidad. Es recomendable la salpingooforectomía unilateral, seguida de una correcta estatificación quirúrgica (citología del líquido peritoneal, biopsia de cualquier área sospechosa peritoneal, muestreo ganglionar paraórtico y pélvico y omentectomía infracólica). Para obtener el mejor beneficio se debe hacer el máximo esfuerzo para quitar toda la enfermedad, ya que, cuanto más completa sea la citorreducción, mejores serán los resultados ${ }^{2}$.

La alfa-fetoproteína es una glicoproteína de 68-70 kDA utilizada como marcador tumoral muy útil para el diagnóstico y seguimiento de mujeres con neoplasias germinales de ovario, se considera elevada si es $>10 \mathrm{ng} / \mathrm{ml}^{3}$.

La función reproductiva después de un teratoma inmaduro de ovario parece ser alentadora, sin embargo, debido a la baja incidencia de esta patología y al pequeño número de casos presentados en los estudios disponibles, no se puede transpolar los resultados a poblaciones mayores. Si bien se conoce el comportamiento de la alfa-fetoproteína posterior a la resección de un teratoma inmaduro, pocos estudios han reportado el comportamiento de la alfa-fetoproteína durante- el embarazo de mujeres con antecedente de teratom $\underline{\bar{a}}$ inmaduro. En el presente reporte presentamos el resültado del embarazo posterior a resección de un teratöma inmaduro tratado con cirugía conservadora y quimioterapia y el comportamiento de alfa-fetoproteína durante el mismo.

\section{Reporte de caso}

Se trata de una mujer de 32 años que ingresó en el Instituto Nacional de Perinatología inicialmente en 2007 por ciclos menstruales irregulares con proiomenorreas y diagnóstico externo de miomatosis utexina. Se encontró ovario derecho con una imagen quística en su interior de alta ecogenicidad, de $1.6 \times 1.9 x$ $1.6 \mathrm{~cm}$, compatible con probable tumor dermoide, con flujos vasculares de alta resistencia y marcadores tumorales normales. Se realizó cistectomía laparoscópica en 2008, con reporte histopatológico de quiste dermoide de ovario derecho. Cinco años después? la paciente inició con distensión abdominal y dolor en el hipogastrio. Se realizó un ultrasonido que reportó:êvario izquierdo con imagen de ecogenicidad mixta, gue alternaba áreas de mayor y menor ecogenicidad sin proyectar sombra acústica, con contornos lobulados, septos gruesos, de $10.6 \times 10.6 \times 10 \mathrm{~cm}$ y volumeñ de $561 \mathrm{cc}$, con vascularidad en septos, compatible con probable teratoma de ovario izquierdo. Se corroboró con tomografía axial computarizada (TAC): masa heterogénea de 12 × $13.5 \times 10.4 \mathrm{~cm}$, y se realizó laparótomía exploradora protocolizada en 2013 con resección de quiste de ovario izquierdo (Fig. 1), con estudio Fransoperatorio reportado como teratoma inmaduro de övario. Se realizó ooforectomía izquierda, linfadenectơmía pélvica derecha e izquierda, resección de omento-subcólico y cúpula subfrénica izquierda, además de miiomectomía de mioma subseroso fúndico y lavado peritoneal, con reporte histopatológico definitivo $\smile$ de teratoma inmaduro de ovario estadio clínico IA (Fig's. 2 y 3). Adicionalmente, se realizó criopreservación de embriones debido a fertilidad no satisfecha, y recibió 


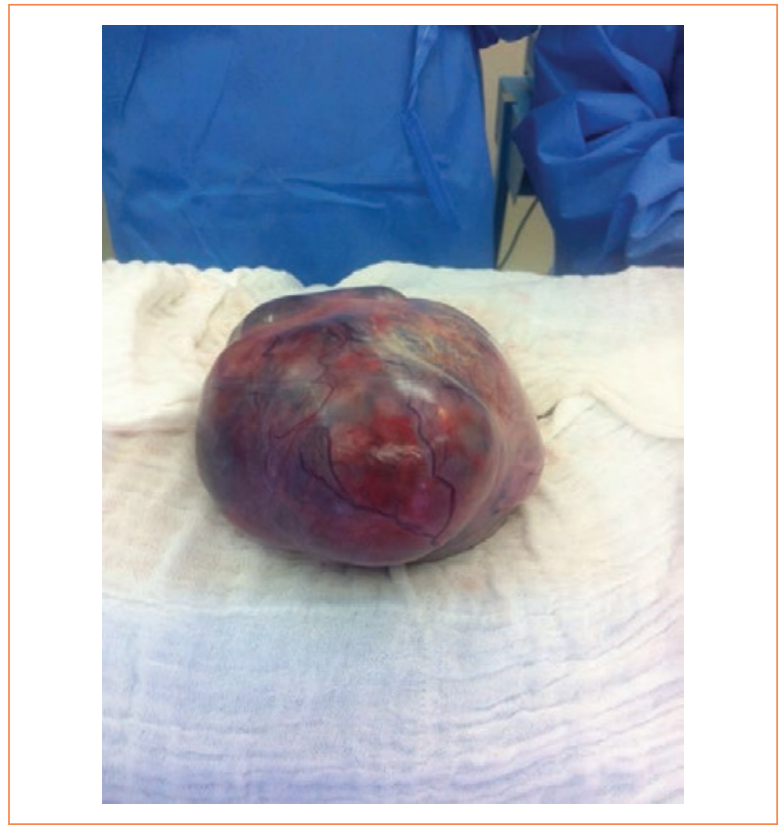

Figura 1. Imagen anatomopatológica correspondiente a una masa heterogénea de 12 × 13.5 x $10.4 \mathrm{~cm}$ en el área correspondiente del ovario izquierdo.

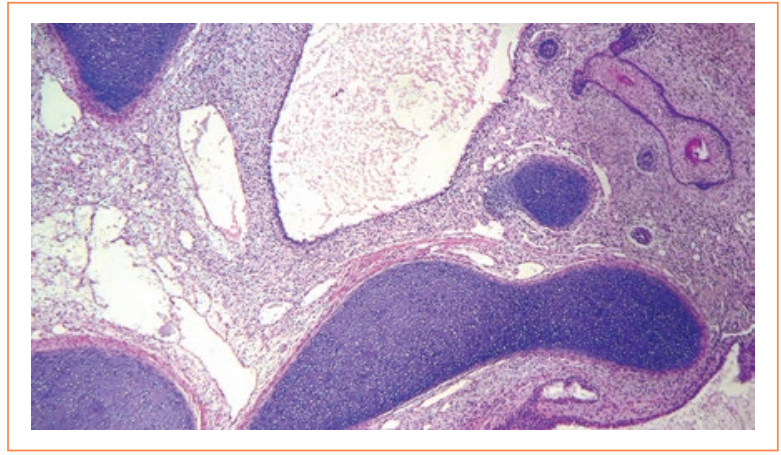

Figura 2. Corte histológico que muestra tejido de aspecto maduro como cartílago, folículos pilosos y epitelio de tipo intestinal, compatible con teratoma inmaduro de ovario.

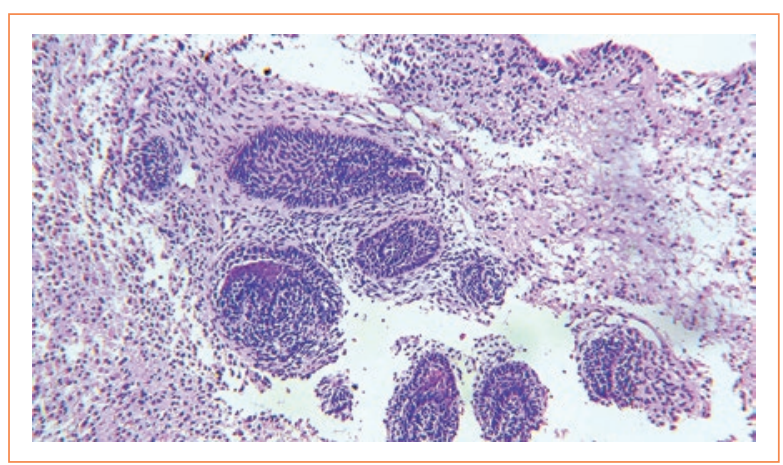

Figura 3. Corte histológico que muestra focos de neuroepitelio dispuestos en pequeños nidos por lo cual se establece clasificarlo como teratoma inmaduro. dos ciclos de quimioterapia adyuvante a base de cisplatino, bleomicina y etopósido.

En 2014 logró un embarazo de forma espontânea. Llevó control prenatal mensual completo, con embarazo normoevolutivo, con vigilancia por los niveles de alfa-fetoproteína (Fig. 4). Se realizó una cesárea ềectiva a las 39 semanas de gestación, y se obtuv@ un recién nacido femenino de $3.565 \mathrm{~kg}$, una talla de $52 \mathrm{~cm}$ y Apgar 9/9. A las 48 horas fue egresada a su domicilio sin complicaciones maternas ni fetales.

\section{Discusión}

En el presente caso se mostró el resultado del embarazo logrado espontáneamente y el comportamientö de la concentración sérica de alfa fetoproteína en una momujer con antecedente de teratoma inmaduro de ovario.

La alfa-fetoproteína se sintetiza en el embarazo pör el saco de Yolk, y conforme el embarazo avanza, el hígado fetal se vuelve el mayor productor, de forma que el pico máximo de producción se alcanza aproximadamente a las 13 semanas de gestación en suero fetal y líquido amniótico, y desciende gradualmente hasta el término. En suero materno, el nivel de alfa fetoproteína se inerementa gradualmente a partir del segundo trimestre, $\bar{c}$ con un nivel promedio de $50 \mathrm{ng} / \mathrm{ml}$ durante el mismo, un pico máximo hacia las 32 semanas de gestación, alcanžando $150 \mathrm{ng} / \mathrm{ml}$, y, posteriormente, un descenso gradual hacia el final del embarazo a un nivel promedio de $50 \mathrm{ng} / \mathrm{ml}^{4}$. En el presente caso se observó una disminución posterior a la ooforectomía, un incremento gradual durante el segundo trimestre, un pico máximo haciąlas 32 semanas de gestación superior al valor esperadő de acuerdo a lo reportado en la literatura, y la disminưueión a valores normales en el puerperio tardío. Si biẹn el riesgo de recurrencia del teratoma es relativamente bajo, la evaluación ovárica durante el segundo y tềcer trimestre del embarazo es difícil y la concentración de alfa fetoproteína podría ser un marcador de seguimiento, sin embargo, la información reportada sobre el comportamiento de los niveles de dicho marcador en mujeres con antecedente de teratoma inmaduro es limitadas.

En el caso presentado, la paciente fue tratada efectivamente de forma conservadora con preservacióñ del ovario contralateral, así como dos ciclos de quimioterapia. La quimioterapia estuvo basada en dos ciclos a base de cisplatino, bleomicina y etopósido. El pronóstico de las mujeres con teratoma inmaduro de ovario depende principalmente del estadio en el que se realice el diagnóstico, así como del grado histológico tumoral ${ }^{5}$. Parā las lesiones de grado 1 , la supervivencia es del $82 \%$; para 


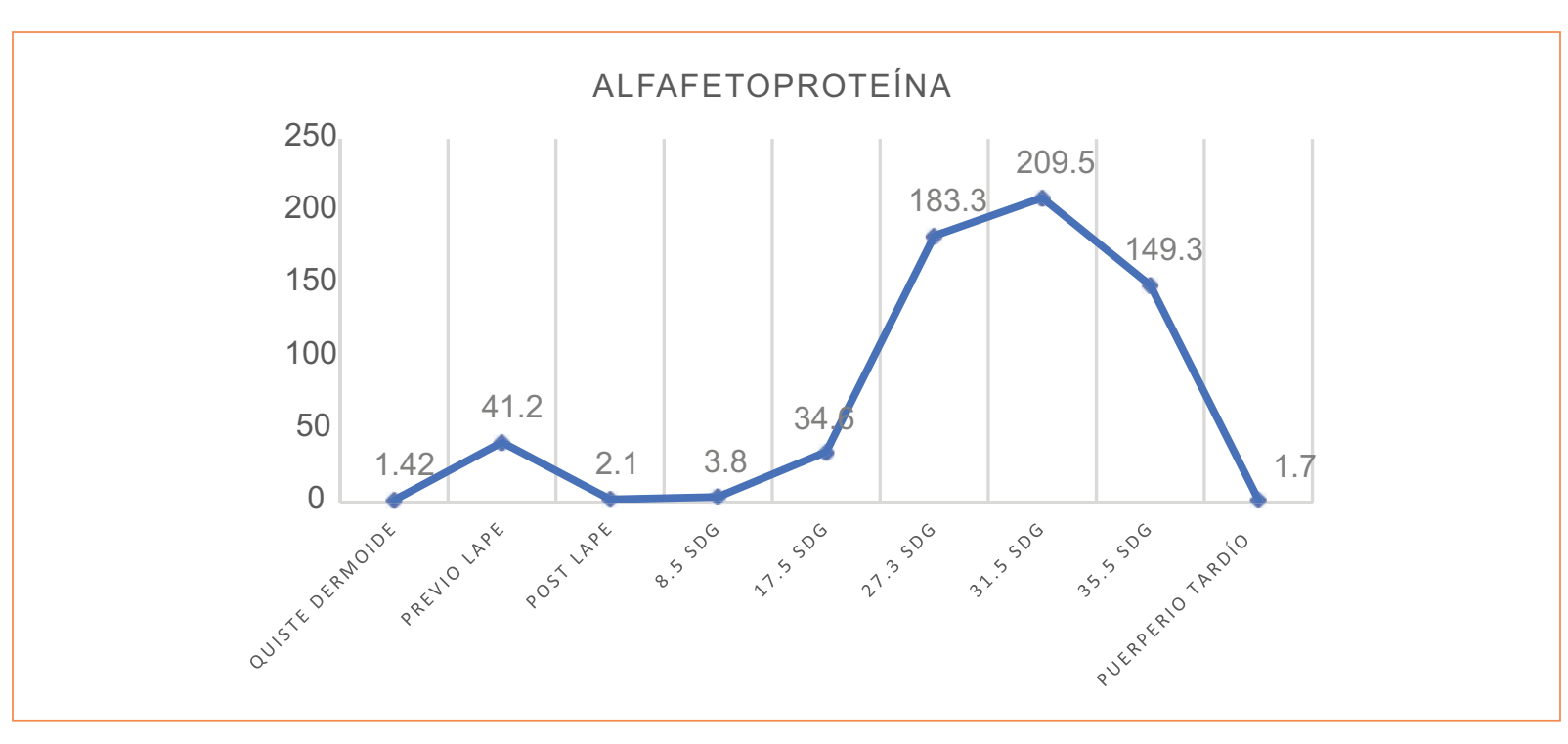

Figura 4. Comportamiento de la concentración de alfa-fetoproteína previo al diagnóstico, posterior al tratamiento durante el embarazo y puerperio.

AFP: alfa-fetoproteína; LAPE: laparotomía exploradora; SDG: semanas de gestación.

las de grado 2 , del $62 \%$, y para las de grado 3 , del $30 \%$. En general, la supervivencia en etapas tempranas (etapas I y II), después de un seguimiento de 39 meses, es del $91.6 \%$ y, en etapas avanzadas (etapa III), la supervivencia llega hasta el $80-85 \%$ en un seguimiento de 4 años, siempre y cuando hayan recibido un adecuado tratamiento quirúrgico y adyuvancia. Para mejores tasas de supervivencia, se debe intentar siempre conseguir una citorreducción óptima y quimioterapia a base de platinos en tumores con grados histológicos o etapa clínica avanzados ${ }^{2}$. La mayoría de las recurrencias ocurren en los primeros dos años después de completar la cirugía. La recurrencia se encuentra típicamente con un aumento de los marcadores tumorales alfa fetoproteína, hormona gonadotropina coriónica y deshidrogenasa láctica o cambios en estudios de imagen ${ }^{5}$.

\section{Conclusiones}

El embarazo posterior a manejo conservador de teratoma de ovario inmaduro puede ser espontáneo y exitoso. En el presente caso, la alfa-fetoproteína se incrementó a partir del segundo trimestre, con un pico máximo en el tercer trimestre, y disminuyó a valores normales en el puerperio tardío.

\section{Financiamiento}

Los autores no recibieron financiamiento para llevar a cabo este artículo.

\section{Conflicto de intereses}

Los autores declaran que no existe conflicto de intereses.

\section{Responsabilidades éticas}

Protección de personas y animales. Los autores declaran que para esta investigación no se han reafizado experimentos en seres humanos ni en animales.

Confidencialidad de los datos. Los autores dečlaran que han seguido los protocolos de su centro de trabajo sobre la publicación de datos de pacientes.

Derecho a la privacidad y consentimiento informado. Los autores han obtenido el consentimiento informado de los pacientes y/o sujetos referidos en el artículo. Este documento obra en poder del autor de correspondencia.

\section{Bibliografía}

1. Alwazzan AB, Popowich S, Dean E, Robinson C, Lotocki R, Altman AD. Pure immature teratoma of the ovary in adults: thirty-year experience of a single tertiary care center. Int J Gynecol Cancer. 2015;25:1616-22.

2. Torres-Lobatón A. Tumores malignos de células germinales del ovario. Estado actual de su diagnóstico y tratamiento. Ginecol Obstet Mex. 2014;82:177-87.

3. El-Bahrawy M. Alpha-fetoprotein-producing non-germ cell tumors of the female genital tract. Eur J Cancer. 2010;46:1317-22.

4. Mizejewski GJ. Levels of alpha-fetoprotein during pregnancy and early infancy in normal and disease states. Obstet Gynecol Surv. ${ }^{2} 2003$; 58:804-26.

5. Reddihalli PV, Subbian A, Umadevi K, Rathod PS, Krishnappa S, Nanaiah SP, et al. Immature teratoma of ovary - outcome following primary and secondary surgery: study of a single institution cohort. Eur J Obstet Gynecol Reprod Biol. 2015;192:17-21. 